\title{
Cosmic String Induced CMB Maps
}

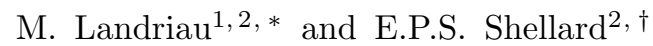 \\ ${ }^{1}$ Max-Planck-Institut für extraterrestrische Physik, Giessenbachstraße 1, 85748 Garching, Germany \\ ${ }^{2}$ Department of Applied Mathematics and Theoretical Physics, \\ University of Cambridge, Centre for Mathematical Sciences, \\ Wilberforce Road, Cambridge CB3 0WA, United Kingdom
}

(Dated: October 30, 2018)

\begin{abstract}
We compute maps of CMB temperature fluctuations seeded by cosmic strings using high resolution simulations of cosmic strings in a Friedmann-Robertson-Walker universe. We create full-sky, $18^{\circ}$ and $3^{\circ} \mathrm{CMB}$ maps, including the relevant string contribution at each resolution from before recombination to today. We extract the angular power spectrum from these maps, demonstrating the importance of recombination effects. We briefly discuss the probability density function of the pixel temperatures, their skewness, and kurtosis.
\end{abstract}

\section{INTRODUCTION}

Despite improving observational limits, interest in cosmic strings has remained durable (for a review, see [1]). Strings are a generic phenomena in fundamental theories and they can emerge in macroscopic form in braneworld cosmologies, for example, at the end of inflation [2, 3]. They are also common to cosmologically viable supersymmetry grand unified theory models [4]. Stringent constraints on strings are important, therefore, in restricting the latitude available for cosmological model building. The detection of cosmic strings would be a watershed for high energy theory.

Despite the potential significance, the investigation of cosmic strings and their observational consequences faces many numerical and analytic challenges, not least in creating accurate realizations of string imprints in the cosmic microwave sky. In this paper, we take this study a step further forward by presenting full-sky and smallangle CMB maps of temperature fluctuations seeded by cosmic string networks using high resolution simulations in an Friedmann-Robertson-Walker expanding universe (with the longest dynamic range to date). This work includes all the relevant recombination physics and can be used not only to determine the angular power spectrum of string CMB anisotropies but also the higher order correlators such as the bispectrum, trispectrum, and beyond.

Current constraints on cosmic strings result from lineof-sight CMB power spectrum calculations sourced either by unequal-time correlators obtained from field theory string simulations [5-7] or semianalytic models of Nambu strings [8, 9]. Qualitatively these two approaches produce consistent spectra, that is, without the strong coherent acoustic peaks associated with inflation. However, quantitatively there is a mismatch between the two approaches in both the shape of the primary peak and its amplitude, which differs by a factor of $2-3$. This dispar-

\footnotetext{
*Email: landriau@mpe.mpg.de

†Email: epss@damtp.cam.ac.uk
}

ity arises primarily from a difference in string network densities, which has been discussed at some length elsewhere [10] (see also [11]). Nevertheless, there is general agreement that the relative amplitude of string induced CMB fluctuations cannot exceed more than $10 \%$ of those arising from adiabatic inflationary perturbations [7, [8]. There have also been a number of studies going beyond the power spectrum through map making with cosmic strings [12 15] in order to study the degree of Gaussianity of the resulting CMB signatures. However, this work has generally only included late-time gravitational effects, ignoring the recombination physics which makes an important contribution to the signal over a wide range of multipoles $l \approx 200-2000$.

The motivation for the present work, then, is twofold: first, to include all recombination effects in the string CMB maps, so that we can ultimately characterize their primary statistical properties, and secondly, to match the accuracy of future experiments such as Planck [16], AMI [17] and QUIET [18] which will impose considerably more stringent constraints on cosmic strings through improving precision, resolution, and added polarization information.

\section{COSMIC STRING NETWORK SIMULATIONS}

Cosmic string simulations were performed with the Allen-Shellard string network code [19]. We have used fixed comoving resolution together with an initial string resolution of 24 points per correlation length. Simulations which started in the matter era had a dynamic range of 7.5 in conformal time, i.e. $\eta_{f}=7.5 \eta_{i}$, but in order to use only the simulation when the network has relaxed into a scaling regime, we ignored the first $4 \%$ of time steps, resulting in an effective dynamic range of 6 in conformal time. Simulations that started in the radiation era had an effective dynamic range of 5 , after eliminating the first $4 \%$ of time steps from a simulation with a dynamic range of 6 . The energy-momentum tensor of the network was projected onto a grid of $256^{3}$ points as described in [20]. The background cosmology used was 
$\Lambda$ CDM with the WMAP 5-year data best fit parameters [21]: $\Omega_{C D M}=0.214, \Omega_{b}=0.044, \Omega_{\Lambda}=0.742$, and $h$ $=0.719$.

We have used three string simulations which span the range from before equality to today. The epochs of each simulation are (1) from $\eta=\eta_{0} / 7.5$ to $\eta_{0},(2)$ from $\eta=\eta_{0} / 45$ to $\eta_{0} / 6$, and (3) from $\eta=\eta_{0} / 216$ to $\eta_{0} / 36$. Thus, these simulations together span a range of 180 in conformal time (note that since we ignore the beginning of each simulation, they do not overlap).

\section{EINSTEIN-BOLTZMANN EVOLUTION}

We use the Landriau-Shellard code [20] to compute cosmological perturbations in Fourier space. Two changes have been made to this code since the algorithm was presented.

The first modification concerns the scalar metric equations employed: Instead of solving for $\dot{h}$ and $\dot{h}^{S}$, we now solve for $\dot{h}$ and $\dot{h}^{-} \equiv \dot{h}-\dot{h}^{S}$, which obeys the following equation:

$$
\ddot{h}^{-}+2 \frac{\dot{a}}{a} \dot{h}^{-}=-16 \pi G(p \Sigma+\delta p)+8 \pi G\left(\Theta+2 \Theta^{S}\right)
$$

The other modification concerns the inverse computation of the evolution equations' fundamental matrices: Instead of LU factorization, we use singular value decomposition, for which we employ the freely available LAPACK routines. This has proved a more numerically stable method and enables a better treatment of near singular matrices [22], especially around recombination.

\section{MAPS}

We compute maps of CMB fluctuations by following photon paths through the simulation boxes. The CMB temperature fluctuations are given by the following equation, obtained by integrating the linear Boltzmann equation for the Stokes parameter $I$ :

$$
\begin{array}{cl}
\frac{\delta T}{T}=\int_{0}^{\eta_{0}} & \left(\dot{\tau} e^{-\tau}\left(\frac{1}{4} \delta_{\gamma}-\mathbf{v}_{\mathbf{B}} \cdot \hat{n}+\frac{1}{4} \Pi_{i j}^{I} \hat{n}_{i} \hat{n}_{j}\right)\right. \\
& \left.-\frac{1}{2} e^{-\tau} \dot{h}_{i j} \hat{n}_{i} \hat{n}_{j}\right) d \eta
\end{array}
$$

where $\Pi_{i j}^{I}$ is the term that couples the Stokes parameter $I$ to $Q$ and $U$ and is given in Fourier space by

$$
\begin{aligned}
\Pi_{i j}^{I}(\mathbf{k}) & =\frac{3}{4}\left(\hat{k}_{i} \hat{k}_{j}-\frac{1}{3} \delta_{i j}\right) \Pi^{S} \\
& -\frac{1}{2}\left(\left(\hat{k}_{i} \hat{e}_{1 j}+\hat{k}_{j} \hat{e}_{1 i}\right) \Pi^{V 1}+\left(\hat{k}_{i} \hat{e}_{2 j}+\hat{k}_{j} \hat{e}_{2 i}\right) \Pi^{V 2}\right) \\
& +\left(\hat{e}_{1 i} \hat{e}_{1 j}-\hat{e}_{2 i} \hat{e}_{2 j}\right) \Pi^{T+}+\left(\hat{e}_{1 i} \hat{e}_{2 j}+\hat{e}_{2 i} \hat{e}_{1 j}\right) \Pi^{T \times}
\end{aligned}
$$

and all the other terms have their usual meaning. A full derivation of this formula is given in [23].
In practice, because $\dot{\tau} e^{-\tau}$ and $e^{-\tau}$ are zero before the start of recombination, we only output grids for all perturbations from $\eta=2 \eta_{\text {rec }} / 3$ to the end of simulation 3 , which is $\eta \simeq 3 \eta_{\text {rec }} / 2$. For simulations 1 and 2 , we only output the grids for $\dot{h}_{i j}$, because $\dot{\tau} e^{-\tau}$ is also zero after the end of recombination.

By putting "observers" at each apex of a cube of side $L / 2$, where $L$ is the simulation box size, we produce eight all-sky maps of resolution $N_{\text {side }}=256$ from simulation 1. From simulation 2 , we compute six $18^{\circ} \times 18^{\circ}$ maps of resolution of $N_{\text {side }}=2048$, by putting an observer outside each face of the simulation box. Finally, from simulation 3, using the same setup as for simulation 2 , we compute six $3^{\circ} \times 3^{\circ}$ maps of $N_{\text {side }}=8192$. Figure 1 1 shows one map produced from each simulation; it should be noted that even the maps of patches of sky are computed using a spherical sky.

Comparing our maps with those of [15], we note that the $18^{\circ}$ map shown and their $7.2^{\circ}$ map have similar features, but the former does not present as sharp linelike discontinuities as the latter. The resolution of our maps is effectively lower than that implied by the Healpix $N_{\text {side }}$ parameter used. This can be seen most directly from the power spectra (see Sec. $(\mathrm{V})$ : Normally, one would expect $\ell_{\max } \lesssim 3 N_{\text {side }}$, but the power in the maps falls around $\ell \approx N_{\text {side }}$, which shows they are over pixelized or, to put it another way, the effective resolution is about a third of that expected from the pixelization. For example, our $18^{\circ}$ maps have an effective resolution of about $5^{\prime}$, compared to $1.7^{\prime}$ expected from a map of $N_{\text {side }}=2048$. This explains the difference in features with the Fraisse et al. maps, which have a resolution of $0.42^{\prime}$, an order of magnitude higher than ours.

\section{POWER SPECTRUM COMPUTATION}

For the all-sky maps, we decompose the temperature fluctuations in the sky in spherical harmonics: $\frac{\delta T}{T}=$ $\sum a_{\ell m} Y_{\ell}^{m}$ and the angular power spectrum is estimated from

$$
C_{\ell}=\frac{1}{2 \ell+1} \sum_{m} a_{\ell m} a_{\ell m}^{*}
$$

For this purpose, we use the Healpix package [24]. For the $3^{\circ}$ patches, we use the flat-sky approximation (see e.g. 25]) which replaces the spherical harmonic transform with a 2-D Fourier transform: $C_{\ell} \simeq C_{k}=\left|a_{\mathbf{k}}\right|^{2}$, where the modes are obtained from $\frac{\delta T}{T}=\sum a_{\mathbf{k}} e^{i \mathbf{k} \cdot \mathbf{x}}$. For the $18^{\circ}$ patches, we have used both methods to compare their relative merit. To use a spherical transform on an incomplete sky, one must multiply the extracted spectrum with a mode decoupling matrix [26]: $C_{\ell}=M_{\ell \ell^{\prime}}^{-1} \tilde{C}_{\ell^{\prime}}$, where

$$
M_{\ell \ell^{\prime}}=\left(2 \ell^{\prime}+1\right) \sum_{L} \frac{(2 L+1)}{4 \pi} C_{L}^{m a s k}\left(\begin{array}{ccc}
\ell & \ell^{\prime} & L \\
0 & 0 & 0
\end{array}\right)^{2},
$$



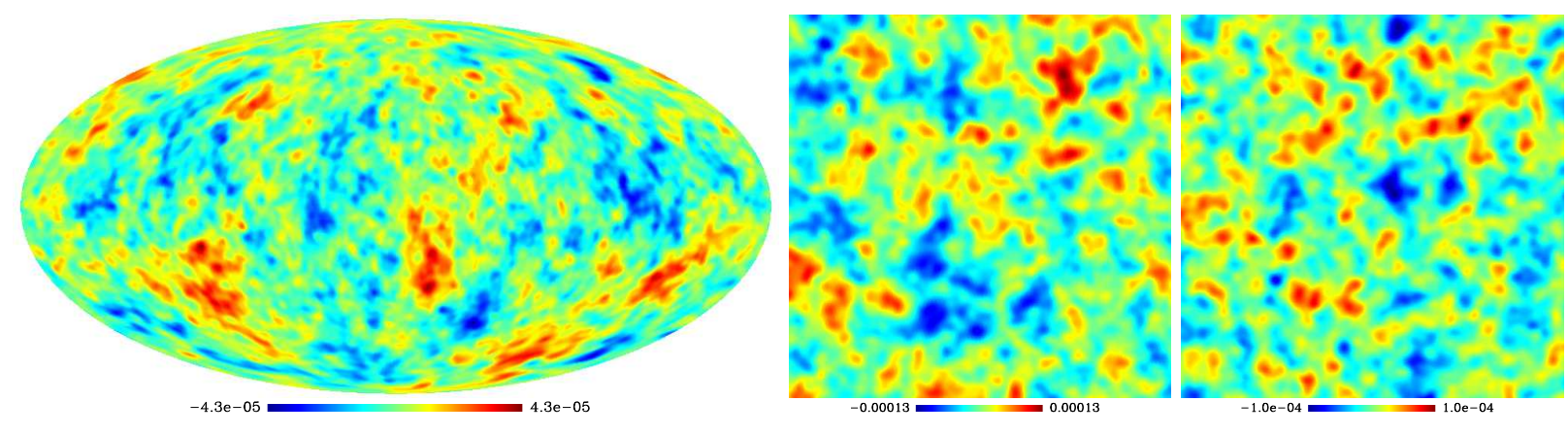

FIG. 1: Temperature fluctuations produced by cosmic strings from simulation 1 (left), simulation 2 (middle), and simulation 3 (right).

However, the resulting spectra show strong oscillations due to the Gibbs effect that are not corrected by the procedure outlined above. Because of this, we have used a 2D Fourier transform even though the flat-sky approximation starts to break down at the lower end of the multipole range probed by these maps. In Fig. 2, we show the angular power spectrum from each of the three simulation sets, as well as their summation (i.e. concatenating these consecutive but nonoverlapping time contributions). For the full sky, all multipoles are shown, while for the patches of sky, the spectra are binned. The errors on the individual parts of the spectrum are estimated by the variance between the maps. To add up the spectra in sections where two sets of maps contribute, we average the lower $l$ part in bins of the same size as that of the higher $l$ part and then add the two contributions. Also, to reduce the error, we binned the part of the spectrum in which only the all-sky maps contribute. We normalize the string spectra using $G \mu / c^{2}=1 \times 10^{-6}$.

In addition, Fig. 3 illustrates the power spectrum from simulation 3 which begins before equal matter radiation and separates out the late-time contribution (dashed line), that is, as if the string simulation and EinsteinBoltzmann evolution were to start just before decoupling $\eta_{\text {dec }}$. Note that maps by other groups have been generated by considering contributions only after decoupling $\eta>\eta_{\text {dec }}$, e.g. [15]. Here, we see starkly illustrated the importance of the early contributions to the CMB anisotropy which arise from matter and radiation perturbations induced by strings before decoupling (primarily scalar modes). For $l>400$, this strong early scalar component predominates over the late-time gravitational string contribution (primarily vector modes). These results contrast markedly with the subdominant scalar power spectra obtained earlier using Nambu string simulations with full Einstein-Boltzmann evolution in a flat cold dark matter model [27]. However, this enhancement is due here to inclusion of the full matter-radiation transition with a significantly higher radiation era string density and a $\Lambda$ concordance cosmology with a higher relative baryon density at decoupling. In future work [28], we shall compare this result with the spectrum obtained from the same simulations' UETCs. The overall shape for the spectrum computed from the $18^{\circ}$ maps is also qualitatively consistent with the CMB power spectrum calculated using a simplified analytic formula [29].

Comparing this result with that of [15] - the only other group to have recently extracted $C_{\ell}$ from simulated maps - we see some significant differences attributable both to the inclusion of recombination physics in our work and to the different approximations used. Firstly, we note that Ref. [15] achieves much better statistics because it is easier to simulate purely gravitational effects in a flatsky approximation at high resolution, generating a larger number of maps which results in smaller error bars. However, for $l \leq 500$, we note that their power spectrum does not appear to turn over in the range $l \lesssim 200-400$, a causality cut-off due to the fact that these string modes are superhorizon at recombination (see e.g. Ref. [29]). In the range $l \approx 400-2000$, as discussed above, there is an important contribution from early-time scalar modes at decoupling which is not included in Ref. [15]. It is reasonable to assume that late-time gravitational effects dominate the spectrum at $l \gg 1000$, and their result in this range is probably fairly accurate. However, in this region we note that the limited spatial resolution of our individual maps (resulting from propagation of photons through strings smoothed onto $256^{3}$ grids) means that there is generically small-scale power missing at higher multipoles from the summed power spectrum in Fig. 2 . For example, the gravitational contribution from strings in simulation 1 (full-sky) is absent beyond $l \approx 100$. For this reason and given the limited number of realizations, we have not yet endeavored to obtain a detailed constraint using WMAP, though we expect it to be consistent with constraints in Ref. [30]. By comparing the Sachs-Wolfe plateau to CMB observations, we find a normalization of the cosmic strings linear energy density to be $G \mu / c^{2}=(1.45 \pm 0.6) \times 10^{-6}$, in agreement with our previous result [14]. 

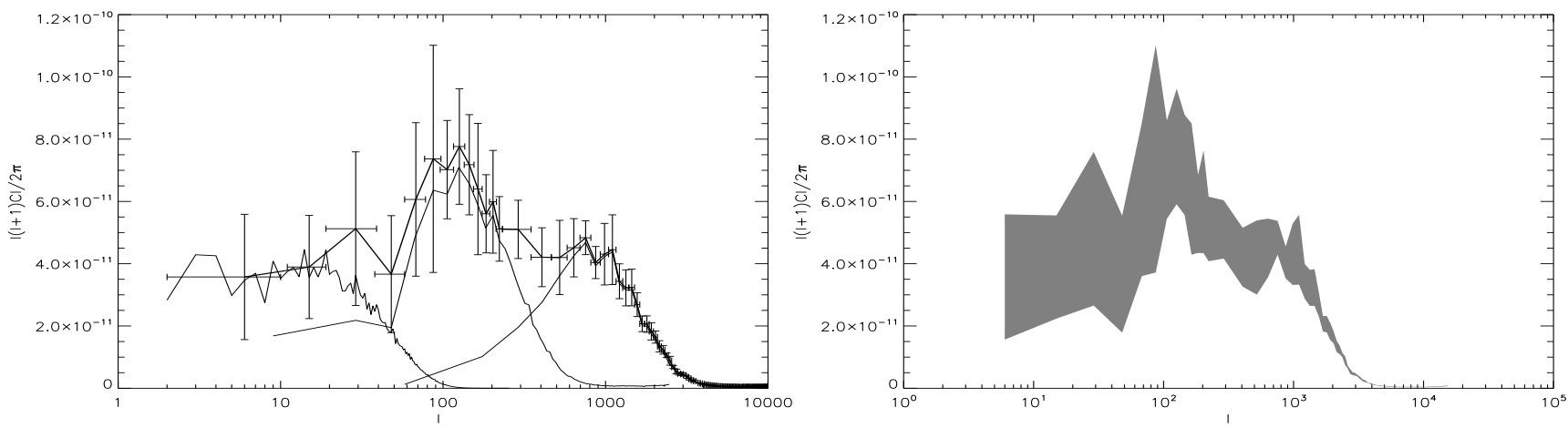

FIG. 2: On the left, we show the angular power spectrum of CMB temperature fluctuations produced by networks of cosmic strings The thin lines represent the spectra from simulations $1-3$ (left to right) and the thick line is their sum. On the right, the total spectrum with its error bars is shown again as an "expectation area."

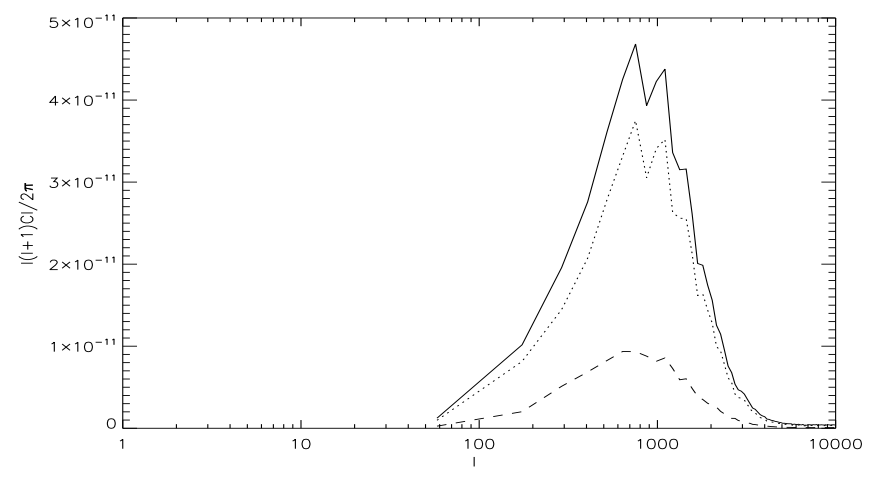

FIG. 3: Power spectrum from simulation 3 using the full simulation (solid line) and the late start evolution (dashed line) and their difference (dotted line).

\section{PROBABILITY DISTRIBUTION FUNCTION}

One of the principal aspects of cosmic string induced CMB fluctuations is their intrinsic non-Gaussianities. In this section, we present preliminary results from our map realizations for the pixel temperature distribution, its skewness and kurtosis. In future work, we shall explore the efficiency of different techniques and tools, such as the bispectrum 31], to better characterize the non-Gaussian signature from strings and thus infer their detectability.

We compute the pixel probability distribution function of the rectangular map pixel grids computed to estimate the power spectrum. To compare our results, we generate an ensemble of 1000 Gaussian maps with the same power spectrum as the string map. To do so, we use the fact that, in Fourier space, a Gaussian field will have phases that are uniformly distributed between 0 and $2 \pi$. Hence, for a given map, after fast Fourier transform, we randomize the phases and fast Fourier transform back to real space. In Fig. (4, we show the temperature distribution for medium- and small-angle maps along with the
Gaussian ensemble for comparison. These probability distribution functions are remarkably Gaussian, consistent with early string map results which demonstrated that, despite the distinct signature of individual strings, the central limit theorem prevailed after many strings contributed [13]. We note, however, that the higher resolution $3^{\circ}$ maps do appear to have a slightly increased level of non-Gaussianity.

We also computed the skewness

$$
\gamma_{1}=\frac{\frac{1}{N_{p i x}} \sum_{i}\left(T_{i}-\bar{T}\right)^{3}}{\sigma^{3}}
$$

and the kurtosis

$$
\gamma_{2}=\frac{\frac{1}{N_{p i x}} \sum_{i}\left(T_{i}-\bar{T}\right)^{4}}{\sigma^{4}}-3
$$

of the string maps and compared it to that of all our Gaussian maps. Figure 5 shows the skewness and kurtosis for all the maps as well as the $1 \sigma$ contour value for the ensemble average of Gaussian realizations. This indicates a marginal positive $1 \sigma$ skewness and kurtosis for the $3^{\circ}$ maps. The absence of a strong skewness in the $18^{\circ}$ maps seems to be at variance with the significant negative skewness $\gamma_{1}=-0.24$ found in the $7.2^{\circ}$ maps from late-time gravitational effects in Ref. [15] (see also the analytic estimates in [29, 32, 33]). Two possible explanations are apparent. First, as mentioned above, the present 3D Einstein-Boltzmann simulations have an effectively lower resolution than the 2D flat-sky approximation maps, so they cannot probe as far into the wings of the distribution. We are missing the integrated effect of bispectrum triangles combining disparate large and small scales. Second, the analytic modeling in Ref. 29] incorporates a causality or correlation-length cut-off which prevents bispectrum and trispectrum contributions from superhorizon scales, effectively flattening their accumulated amplitude below $l \approx 300-500$. Without such a cut-off introduced through energy-momentum compensation, there may be a significant enhancement of the skewness. 

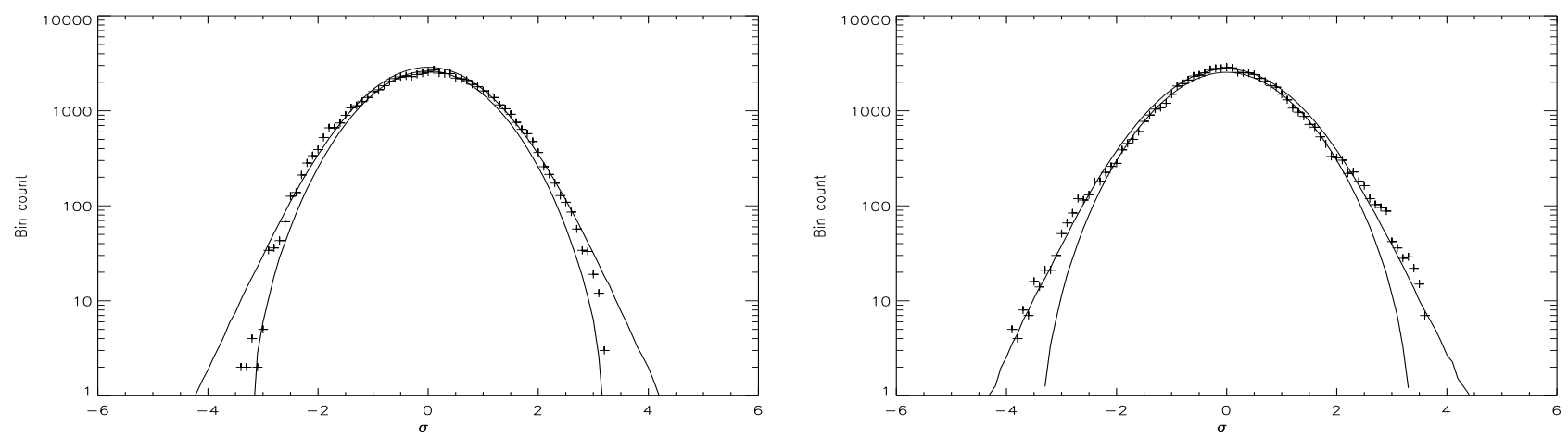

FIG. 4: Temperature distributions of the medium- (left) and small- (right) angle maps shown above. The solid lines indicate the $1 \sigma$ level of an ensemble of Gaussian maps

The tentative indications of positive skewness and kurtosis in the $3^{\circ}$ maps, however, are consistent with physical expectations. On scales above $l>300$, we see that there is an important contribution from early scalars with strings generating wakelike objects in the matter distribution with a positive skewness and kurtosis [34]. This will lead to CMB anisotropies with similar properties. It appears that this positive skewness will compete with and confuse the negative skewness predicted for the latetime gravitational effects on intermediate scales. In contrast, the kurtosis of these two contributions will sum positively, reinforcing the view that the trispectrum will prove a better discriminant of strings than the bispectrum [29]. These preliminary results indicate that incorporating recombination effects will be important in any effort to constrain non-Gaussian strings signatures with WMAP or Planck data.

\section{CONCLUSION}

We have computed maps of cosmic string induced CMB fluctuations at various resolutions and we have ex- tracted their angular power spectra. We have demonstrated the importance of recombination effects for the power spectrum over a broad range of multipoles $200<$ $l<2000$. We have also shown that the resulting maps are remarkably Gaussian, though with potential deviations which are worthy of closer investigation as testable string signatures in the CMB.

\section{Acknowledgments}

Numerical simulations were performed on COSMOS, the SGI Altix 4700, owned by the United Kingdom Cosmology Consortium, funded by STFC, SGI and Intel. We were also supported by STFC Grant No. ST/P002998/1. We are very grateful to Donough Regan, Carlos Martins, and Eiichiro Komatsu for useful discussions about this work. We thank Michele Liguori for his code to generate Wigner $3 \mathrm{j}$ symbols.
[1] A. Vilenkin and E. P. S. Shellard, Cosmic Strings and other Topological Defects (Cambridge University Press, Cambridge, England, 2000).

[2] S. Sarangi and S.-H. H. Tye, Phys. Lett. B536, 185 (2002).

[3] E. J. Copeland, R. C. Myers, and J. Polchinski, JHEP 0406, 013 (2004).

[4] R. Jeannerot, J. Rocher, and M. Sakellariadou, Phys. Rev. D68, 103514 (2003).

[5] U. L. Pen, U. Seljak, and N. Turok, Phys. Rev. Lett. 79, 1611 (1997).

[6] R. Durrer, M. Kunz, and A. Melchiorri, Phys. Rev. D59, 123005 (1999).

[7] N. Bevis, M. Hindmarsh, and M. Kunz, Phys. Rev. D70, 043508 (2004).
[8] R. A. Battye, J. Robinson, and A. Albrecht, Phys. Rev. Lett. 80, 4847 (1998).

[9] L. Pogosian and T. Vachaspati, Phys. Rev. D60, 083504 (1999).

[10] C. J. A. P. Martins, J. N. Moore, and E. P. S. Shellard, Phys. Rev. Lett. 92, 251601 (2004).

[11] R. A. Battye, B. Garbrecht, A. Moss, and H. Stoica, JCAP 0801, 020 (2008).

[12] D. P. Bennett and F. R. Bouchet, Phys. Rev. Lett. 60, 257 (1988).

[13] B. Allen, R. R. Caldwell, E. P. S. Shellard, A. Stebbins, and S. Veeraraghavan, Phys. Rev. Lett. 77, 3061 (1996).

[14] M. Landriau and E. P. S. Shellard, Phys. Rev. D69, 023003 (2004), we note that the normalisation is different due to the value of $k$-modes differing by a factor of $\sqrt{3}$. 

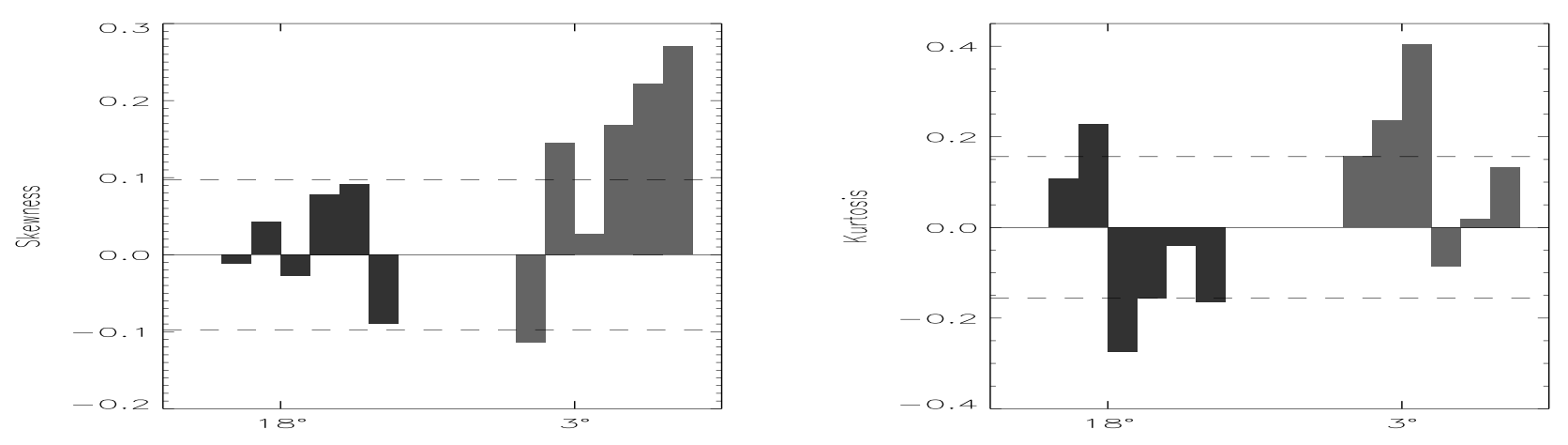

FIG. 5: Skewness (left) and kurtosis (right) for all six $18^{\circ}$ and $3^{\circ}$ maps. The lines show the $1 \sigma$ value for the ensemble of Gaussian maps.

[15] A. A. Fraisse, C. Ringeval, D. N. Spergel, and F. R. Bouchet, Phys. Rev. D78, 043535 (2008).

[16] The Planck Consortium, Tech. Rep. ESA-SCI(2005)1, European Space Agency (2005).

[17] J. T. L. Zwart et al., MNRAS 391, 1545 (2008).

[18] D. Samtleben, Nuovo Cimento B Serie 122, 1353 (2007).

[19] B. Allen and E. P. S. Shellard, Phys. Rev. Lett. 64, 119 (1990).

[20] M. Landriau and E. P. S. Shellard, Phys. Rev. D67, 103512 (2003).

[21] J. Dunkley et al., ApJ S180, 306 (2009).

[22] W. H. Press, S. A. Teukolsky, W. T. Vetterling, and B. P. Flannery, Numerical Recipes in C, The Art of Scientific Computing (Cambridge University Press, Cambridge, England, 1992), 2nd ed.

[23] M. Landriau, E. P. S. . Shellard, and E. Komatsu, in preparation.

[24] K. M. Górski, E. Hivon, A. J. Banday, B. D. Wandelt, F. K. Hansen, M. Reinecke, and M. Bartelmann, ApJ 622, 759 (2005).

[25] M. White, J. E. Carlstrom, M. Dragovan, and W. L.
Holzapfel, ApJ 514, 12 (1999).

[26] E. Hivon, K. M. Górski, C. B. Netterfield, B. P. Crill, S. Prunet, and F. Hansen, Astrophys. J. 567, 2 (2002).

[27] B. Allen, R. R. Caldwell, S. Dodelson, L. Knox, E. P. S. Shellard, and A. Stebbins, Phys. Rev. Lett. 79, 2624 (1997).

[28] M. Landriau, C. J. A. P. Martins, and E. P. S. Shellard, in preparation.

[29] D. M. Regan and E. P. S. Shellard, Phys. Rev. D 82, 063527 (2010).

[30] R. Battye, B. Garbrecht, and A. Moss, Phys. Rev. D 81, 123512 (2010).

[31] J. Fergusson, M. Landriau, M. Liguori, and E. P. S. Shellard, in preparation.

[32] M. Hindmarsh, C. Ringeval, and T. Suyama, Phys. Rev. D 80, 083501 (2009).

[33] D. Yamauchi et al., JCAP 05, 033 (2010).

[34] P. P. Avelino, E. P. S. Shellard, J. H. P. Wu, and B. Allen, ApJ Lett 507, L101 (1998). 